\title{
Observer-Based Adaptive Neural Control for a Class of Nonlinear Non-affine Systems with Unknown Gain Sign
}

\author{
Mohammad M. Arefi, Mohammad R. Jahed-Motlagh \\ Iran University of Science and Technology, Tehran 16846-13114, Iran. \\ e-mail :\{arefi,jahedmr\}@iust.ac.ir
}

\begin{abstract}
This paper presents an adaptive neural network output feedback controller for a class of uncertain SISO nonlinear non-affine systems. Since the system states are not required to be available for measurement, an observer is designed to estimate the system states. Comparing to existing results, this method does not require a priori knowledge about the sign of control gain direction. To deal with the unknown sign of the control gain, the Nussbaum-type function is used. By using neural network, the unknown nonlinear function is approximated and a robustifying term is used to reduce the approximation error and compensate the effect of external disturbance. The stability of the closed-loop system is analyzed by using Lyapunov method. Theoretical results are illustrated through simulation example. Numerical simulations confirm the effectiveness of the proposed method.
\end{abstract}

Keywords: Adaptive control, Neural network control, non-affine nonlinear systems, Observer design, Nussbaum function.

\section{INTRODUCTION}

Robust control of uncertain nonlinear systems is a challenging problem in control theory and has attracted a lot of attention in the last three decades (Lian et al. 2009). However, some of the previous results are based on the assumption that the mathematical model of the nonlinear system is known (Khalil 2002). On the other hand, it is difficult to exploit mathematical models of many systems in the real world. In this case, the conventional control methods may not be used and it is desirable to use the adaptive approach based on universal functions approximator (UFA) (Khalil 2002; Du et al. 2009; Liu and Zheng 2009a; Frikha et al. 2010; Boulkroune et al. 2010; Leu et al. 1999; Liu and Zheng 2009b; Liu and Li; Liu et al. 2009; Li et al. 2003).

In the last decade the analytical study of adaptive control of uncertain nonlinear systems using UFA has received much attention. Typically, these methods utilize neural network $(\mathrm{NN})$ and fuzzy logic (FL) as approximation models for the unknown uncertainties. After that, the controller is designed by adaptive laws for adjusting the parameters of the UFAs. Conceptually, there are two distinct approaches to design an adaptive controller based on UFAs: direct and indirect approaches (Liu et al. 2009; Li et al. 2003; Labiod and Guerra 2007a; Ordonez et al. 1999; Shaocheng et al. 2005). In indirect adaptive control schemes, the main effort is to avoid the possible singularity problem when calculating the inverse of the estimated decoupling matrix (i.e. the estimate of the control gain matrix) (Shaocheng et al. 2005). To deal with singularity problem some methods are proposed in literatures. For example, the projection algorithm is widely used to keep the estimated parameter of control gain matrix inside a feasible set in which the singularity problem does not happen (Leu et al. 1999; Chang 2000). However, this method usually needs a priori knowledge for the feasible parameters set and there is not a systematic procedure for constructing this feasible set in general. Another approach for overcoming singularity problem is to use regularized inverse of control gain matrix instead of its inverse in the control law (Labiod and Guerra 2007a; Shaocheng et al. 2005). In this paper, an indirect adaptive neural network controller is suggested to solve this problem.

A common restriction in the UFAs-based adaptive control is that it is required to assume the sign of the control gain matrix is known. Nevertheless, this assumption is not realistic in general case i.e. the sign of control gain matrix is often unknown and called an unknown control direction or unknown high-frequency gain (Ye 2001). When there is not a priori knowledge about the sign of control gain, the problem becomes much more difficult. The sound solution of this problem is to use Nussbaum-gain technique (Nussbaum 1983). The adaptive fuzzy state feedback control of a class of uncertain MIMO nonlinear systems with unknown control gain matrix has been proposed in (Boulkroune et al. 2010). However, most of the previous results are based on the assumption that state variables of the system are available. If the system states are unavailable, these results are not applicable in practice and the UAF-based adaptive control using estimated states is then required (Du et al. 2009; Frikha et al. 2010; Leu et al. 1999; Liu and Zheng 2009b; Liu et al. 2009; Li et al. 2003). In ( $\mathrm{Liu}$ and Li), the adaptive fuzzy output feedback control for a class of uncertain SISO 
nonlinear with unknown control gain has been presented. In recent years, much progress has been made in the field of adaptive fuzzy control for uncertain nonlinear non-affine systems (Labiod and Guerra 2007b; Park et al. 2005). In these approaches, the mean value theory is firstly used to transform unknown non-affine functions into the structure which is similar to affine form, but there are still some nonaffine functions in this structure. A common assumption is that the sign of the control gain coefficient in the transformed systems is known (Du et al. 2009; Labiod and Guerra 2007b; Park et al. 2005). In (Liu and Wang 2009), the adaptive fuzzy state feedback control of a class of uncertain SISO non-affine nonlinear system with unknown control gain is presented. In this paper, observer-based adaptive neural network control of a class of uncertain nonaffine nonlinear systems with unknown control direction is developed. The main contributions of this work can be summarized as follows:

(i) This paper successfully extends the Nussbaum-gain technique to a SISO non-affine system with unmeasured states.

(ii) The controller singularity problem is avoided by using mean-value theorem and an indirect neural network controller.

(iii) By using a robustifying term in the control signal, the effects of approximation error in neural network and external disturbance is compensated.

The rest of this paper is organized as follows: The problem is formulated in Section 2. In Section 3, we present a procedure to design the observer-based adaptive neural network controller. In Section 4, simulation results are presented to confirm the effectiveness of the proposed method. Finally, the conclusions are given in section 5 .

\section{PROBLEM FORMULATION}

Assume that a nonlinear SISO system is represented as follows:

$$
\begin{aligned}
& \dot{x}_{i}=x_{i+1}, \quad i=1,2, \ldots, n-1 \\
& \dot{x}_{n}=f(\mathbf{x}, u)+d(t) \\
& y=x_{1}
\end{aligned}
$$

where $\mathbf{x}=\left[x_{1}, \ldots, x_{n}\right]^{\mathrm{T}}$ is the plant state vector, $u$ is the control input of the system, $y$ is the output, $f(\mathbf{x}, u)$ is an unknown smooth non-affine function in control, and $d(t)$ is the external disturbance. The control objective is that the system output can track reference signal $y_{m}$. We make the following assumption regarding the system (1).

Assumption 1. The function $g(\mathbf{x}, u)=\frac{\partial f(\mathbf{x}, u)}{\partial u} \neq 0$ is nonzero for all $(\mathbf{x}, u) \in \Omega_{\mathbf{x}} \times \mathfrak{R}$ with a controllability region $\Omega_{\mathbf{x}}$.

Assumption 2. A trajectory $y_{m}$ and its $n$ derivatives, namely $y_{m}^{i}, i=1, \ldots, n$, are smooth, bounded and available for design.
Remark 1. A common assumption in (Labiod and Guerra 2007b; Park et al. 2005) is that the function $g(\mathbf{x}, u)$ is either strictly positive or negative which means the gain sign should be known in advance. However, Assumption 1 implies that the gain sign of $g(\mathbf{x}, u)$ does not require to be known. In this paper, the Nussbaum-gain technique is used to deal with this problem.

Definition 1 (Nussbaum 1983) A function is $N(\varsigma)$ called Nussbaum type function if it has the following properties:

$$
\begin{aligned}
& \lim _{z \rightarrow \infty} \sup \frac{1}{z} \int_{0}^{z} N(\varsigma) d \varsigma=+\infty \\
& \lim _{z \rightarrow \infty} \inf \frac{1}{z} \int_{0}^{z} N(\varsigma) d \varsigma=-\infty
\end{aligned}
$$

Commonly used Nussbaum functions are:

$\varsigma^{2} \cos (\varsigma), \varsigma^{2} \sin (\varsigma)$ and $\exp \left(\varsigma^{2}\right) \cos \left(\frac{\pi \varsigma}{2}\right)$. Throughout this paper the even Nussbaum function $N(\varsigma)=\exp \left(\varsigma^{2}\right) \cos \left(\frac{\pi \varsigma}{2}\right)$ is employed.

Lemma 1. (Xudong et al. 1998). Let $\mathrm{V}(t), \varsigma(t)$ be functions defined on $\left[0, t_{f}\right)$ with $\mathrm{V}(t)>0, \forall t \in\left[0, t_{f}\right)$, and $N(\varsigma)$ be an even smooth Nussbaum-type function. If the following inequality holds:

$\mathrm{V}(t) \leq c_{0} \pm \int_{0}^{t}(g N(\varsigma) \pm 1) \dot{\zeta} d \tau$

where $c_{0}$ represents some suitable constant, and $g$ is a nonzero constant, then $\mathrm{V}(t), \varsigma(t)$, and $\int_{0}^{t}(g N(\varsigma) \pm 1) \dot{\zeta} d \tau$ must be bounded on $\left[0, t_{f}\right)$.

Lemma 2. (Mean Value Theorem). Assume that $f(x, y): \mathfrak{R}^{n} \times \mathfrak{R} \rightarrow \mathfrak{R}$ has a derivative at each point of an open set $\mathfrak{R}^{n} \times(a, b)$, and assume also that it is continuous at both endpoints $y=a$ and $y=b$. Then, there is a point $\pi \in(a, b)$ such that (Du et al. 2009)

$f(x, b)-f(x, a)=f^{\prime}(x, \pi)(b-a)$

Define the tracking error as

$e_{1}=y-y_{m}$

Denote

$\mathbf{x}=\left[x_{1}, \ldots, x_{1}^{(n-1)}\right]^{\mathrm{T}}$

$\mathbf{y}_{m}=\left[y_{m}, \ldots, y_{m}^{(n-1)}\right]^{T}$

then

$\mathbf{e}=\mathbf{x}-\mathbf{y}_{m}=\left[e_{1}, \ldots, e_{1}^{n-1}\right]^{\mathrm{T}}$

Since $\dot{\mathbf{y}}_{m}=\mathbf{A} \mathbf{y}_{m}+\mathbf{B} y_{m}^{n}$, it is obtained that

$\dot{\mathbf{e}}=\mathbf{A e}+\mathbf{B}\left[f(\mathbf{x}, u)-y_{m}^{n}+d(t)\right]$

$e_{1}=\mathbf{C}^{\mathrm{T}} \mathbf{e}$ 
where

$\mathbf{A}=\left[\begin{array}{cccc}0 & 1 & \ldots & 0 \\ 0 & 0 & \ldots & 0 \\ \vdots & \vdots & \ldots & \vdots \\ 0 & 0 & \ldots & 1 \\ 0 & 0 & \ldots & 0\end{array}\right]_{n \times n}$

$\mathbf{B}=\left[\begin{array}{c}0 \\ 0 \\ \vdots \\ 0 \\ 1\end{array}\right]_{n \times 1}, \mathbf{C}^{\mathrm{T}}=[1,0, \ldots, 0]_{1 \times n}$

Using the mean value theorem, we have

$f(x, u)=f(\mathbf{x})+g\left(\mathbf{x}, u^{*}\right) u$

where $u^{*}$ is some point between zero and $u$. Then, (9) becomes:

$\dot{\mathbf{e}}=\mathbf{A e}+\mathbf{B}\left[f(\mathbf{x})+g\left(\mathbf{x}, u^{*}\right) u-y_{m}^{n}+d(t)\right]$

$e_{1}=\mathbf{C}^{\mathrm{T}} \mathbf{e}$

Based on the certainty equivalence approach, when $f(\mathbf{x})$, $g\left(\mathbf{x}, u^{*}\right)$ are known, $d(t)=\mathbf{0}$, and the state $\mathbf{x}$ are available, from (11) the ideal controller can be chosen as

$u_{\text {ideal }}=g^{-1}\left(\mathbf{x}, u^{*}\right)\left[-f(\mathbf{x})+y_{m}^{n}-\mathbf{K}_{\mathrm{c}}^{\mathrm{T}} \mathbf{e}\right]$

where $\mathbf{K}_{\mathrm{c}}^{\mathrm{T}}$ is the feedback gain vector to make the characteristic polynomial of $\mathbf{A}-\mathbf{B K}_{\mathrm{c}}^{\mathrm{T}}$ Hurwitz. Thus, it can be obtained that $\lim _{t \rightarrow \infty} \mathbf{e}(t)=\mathbf{0}$. However, $f(\mathbf{x})$ and $g\left(\mathbf{x}, u^{*}\right)$ are unknown and the systems state $\mathbf{x}$ is unmeasurable, so the ideal controller $u_{\text {ideal }}$ cannot be implemented. A sound solution is to use NNs to approximate unknown functions and design an observer to estimate the system state $\mathbf{x}$. It has been proven that the $\mathrm{NN}$ can uniformly approximate nonlinear continuous functions to an arbitrary accuracy.

\section{OBSERVER-BASED INDIRECT ADAPTIVE NEURAL CONTROL}

Since the state vector $\mathbf{x}$ is assumed to be unmeasurable, it cannot be used in the controller design. Therefore an observer should be designed to estimate the unmeasurable states. Consider the following observer:

$\dot{\hat{\mathbf{e}}}=\mathbf{A} \hat{\mathbf{e}}-\mathbf{B} \mathbf{K}_{\mathrm{c}}^{\mathrm{T}} \hat{\mathbf{e}}+\mathbf{K}_{o}\left(e_{1}-\hat{e}_{1}\right)$

$\hat{e}_{1}=\mathbf{C}^{\mathrm{T}} \hat{\mathrm{e}}$

where $\mathbf{K}_{o}$ is the observer gain vector to make sure that the characteristic polynomial $\mathbf{A}-\mathbf{K}_{o} \mathbf{C}^{\mathrm{T}}$ is Hurwitz and $\hat{\mathbf{e}}$ is the estimate of $\mathbf{e}$. Define the observation error as $\widetilde{\mathbf{e}}=\mathbf{e}-\hat{\mathbf{e}}$ and using (11) and (12) we have

$\dot{\tilde{\mathbf{e}}}=\left(\mathbf{A}-\mathbf{K}_{o} \mathbf{C}^{\mathrm{T}}\right) \widetilde{\mathbf{e}}+\mathbf{B}\left[f(\mathbf{x})-y_{m}^{n}+\mathbf{K}_{\mathrm{c}}^{\mathrm{T}} \hat{\mathbf{e}}+d(t)+g\left(\mathbf{x}, u^{*}\right) u\right]$

$\widetilde{e}_{1}=\mathbf{C}^{\mathrm{T}} \widetilde{\mathbf{e}}$
Equation (13) can be written as

$$
\begin{aligned}
\dot{\widetilde{\mathbf{e}}}= & \left(\mathbf{A}-\mathbf{K}_{o} \mathbf{C}^{\mathrm{T}}\right) \widetilde{\mathbf{e}}+\mathbf{B}\left[f(\mathbf{x})-y_{m}^{n}+\mathbf{K}_{\mathrm{c}}^{\mathrm{T}} \hat{\mathbf{e}}+d(t)+\right. \\
& \left.\left(g\left(\mathbf{x}, u^{*}\right)+g_{0}\right) u-g_{0} u\right] \\
\widetilde{e}_{1}= & \mathbf{C}^{\mathrm{T}} \widetilde{\mathbf{e}}
\end{aligned}
$$

where $g_{0}$ is an arbitrary nonzero constant.

Posing $f_{1}(\overline{\mathbf{x}})=f(\mathbf{x})+\left(g\left(\mathbf{x}, u^{*}\right)+g_{0}\right) u, \quad$ equation becomes

$\dot{\widetilde{\mathbf{e}}}=\left(\mathbf{A}-\mathbf{K}_{o} \mathbf{C}^{\mathrm{T}}\right) \widetilde{\mathbf{e}}+\mathbf{B}\left[f_{1}(\overline{\mathbf{x}})-y_{m}^{n}+\mathbf{K}_{\mathrm{c}}^{\mathrm{T}} \hat{\mathbf{e}}+d(t)-g_{0} u\right]$

$\widetilde{e}_{1}=\mathbf{C}^{\mathrm{T}} \widetilde{\mathbf{e}}$

where $\overline{\mathbf{x}}=\left[\mathbf{x}^{\mathrm{T}}, u\right]^{\mathrm{T}}$. Thereafter, the neural estimate of $f_{1}(\overline{\mathbf{x}})$ is used to develop an adaptive controller with its adaptation law to meet control objective and guarantee boundedness of all signals in the closed-loop system. Since $\widetilde{e}_{1}$ in equation (15) is available, we will use the strict-positive-real (SPR) Lyapunov design approach to design the stability of the closed-loop system. The output error dynamics of (15) can be given as

$\widetilde{e}_{1}=H(s)\left[f_{1}(\overline{\mathbf{x}})-y_{m}^{n}+\mathbf{K}_{\mathrm{c}}^{\mathrm{T}} \hat{\mathbf{e}}+d(t)-g_{0} u\right]$

where

$H(s)=\mathbf{C}^{\mathrm{T}}\left(s \mathbf{I}-\left(\mathbf{A}-\mathbf{K}_{o} \mathbf{C}^{\mathrm{T}}\right)\right)^{-1} \mathbf{B}$

The transfer function $\mathbf{H}(s)$ is a known stable transfer function. In order to use the SPR-Lyapunov design approach, (16) can be written as

$$
\begin{gathered}
\widetilde{e}_{1}=H(s) L(s)\left[L^{-1}(s) f_{1}(\overline{\mathbf{x}})+L^{-1}(s)\left(\mathbf{K}_{\mathrm{c}}^{\mathrm{T}} \hat{\mathbf{e}}-y_{m}^{n}\right)+\right. \\
\left.L^{-1}(s) d(t)-L^{-1}(s) g_{0} u\right]
\end{gathered}
$$

with

$L(s)=s^{m}+b_{1} s^{m-1}+\cdots+b_{m}(m<n)$

$L(s)$ is chosen so that $L^{-1}(s)$ is a proper stable transfer function and $H(s) L(s)$ is a proper SPR transfer function. Then the state-space realization of (18) can be expressed as

$$
\begin{aligned}
\dot{\tilde{\mathbf{e}}}= & \left(\mathbf{A}-\mathbf{K}_{o} \mathbf{C}^{\mathrm{T}}\right) \widetilde{\mathbf{e}}+\mathbf{B}_{c}\left[L^{-1}(s) f_{1}(\overline{\mathbf{x}})+L^{-1}(s)\left(\mathbf{K}_{\mathrm{c}}^{\mathrm{T}} \hat{\mathbf{e}}-y_{m}^{n}\right)+\right. \\
& \left.L^{-1}(s) d(t)-L^{-1}(s) g_{0} u\right]
\end{aligned}
$$

$\widetilde{e}_{1}=\mathbf{C}^{\mathrm{T}} \widetilde{\mathbf{e}}$

with the following SPR transfer function

$$
H_{c}(s)=\mathbf{C}^{\mathrm{T}}\left(s \mathbf{I}-\left(\mathbf{A}-\mathbf{K}_{o} \mathbf{C}^{\mathrm{T}}\right)\right)^{-1} \mathbf{B}_{c}
$$

Remark 2. The choice of vector $\overline{\mathbf{x}}$ (input arguments of unknown function $\left.f_{1}(\overline{\mathbf{x}})\right)$ is not unique. In fact, since we know that $u$ is function of states $\mathbf{x}$ and $\mathbf{y}_{m}^{n}$, and also $\mathbf{y}_{m}^{n}$ is bounded, then it can be seen quite simply that $\overline{\mathbf{x}}$ are function of $\mathbf{x}$.

By using approximation property of NNs, unknown function $f_{1}(\overline{\mathbf{x}})$ can be approximated by the following two layer RBF neural network with $l$ nodes: 


$$
\hat{f}_{1}(\hat{\mathbf{x}} \mid \boldsymbol{\Theta})=\boldsymbol{\Theta} \xi(\hat{\mathbf{x}})
$$

where

$\boldsymbol{\Theta}=\left[\begin{array}{llll}\theta_{11} & \theta_{12} & \cdots & \theta_{11}\end{array}\right]$ is the adjustable parameter vector of the neural network system, and $\xi(\hat{\mathbf{x}})=\left[\xi_{1}(\hat{\mathbf{x}}), \ldots, \xi_{l}(\hat{\mathbf{x}})\right]^{\mathrm{T}}$ is a neural basis function vector which is fixed a priori by the designer. The basis function $\xi_{i}(\hat{\mathbf{x}})$ is chosen as the commonly used Gaussian functions

$\xi_{i}(\mathbf{x})=\exp \left(-\frac{\left\|\mathbf{x}-\boldsymbol{\mu}_{i}\right\|^{2}}{\eta_{i}^{2}}\right), \quad i=1,2, \ldots, l$

where $\boldsymbol{\mu}_{i}=\left[\mu_{i 1}, \mu_{i 1}, \ldots, \mu_{i n}\right]^{\mathrm{T}}$ and $\eta_{i}$ are the center and the width of the Gaussian function respectively.

It is assumed that $\mathbf{x}, \hat{\mathbf{x}}$, and $\boldsymbol{\Theta}$ belong to compact sets $\mathrm{U}_{1}, \mathrm{U}_{2}$, and $\Omega$, respectively, which are defined as

$$
\begin{aligned}
& \mathrm{U}_{1}=\left\{\mathbf{x} \in \mathfrak{R}^{n}:\|\mathbf{x}\| \leq \mathrm{M}_{1}\right\} \\
& \mathrm{U}_{2}=\left\{\hat{\mathrm{x}} \in \mathfrak{R}^{n}:\|\hat{\mathbf{x}}\| \leq \mathrm{M}_{2}\right\} \\
& \Omega=\left\{\boldsymbol{\Theta} \in \mathfrak{R}^{l}:\|\boldsymbol{\Theta}\| \leq \mathrm{M}_{3}\right\}
\end{aligned}
$$

where $\mathrm{M}_{1}, \mathrm{M}_{2}$, and $\mathrm{M}_{3}$ are the designed parameters.

Define the optimal parameter vector $\mathbf{\Theta}^{*}$

$$
\boldsymbol{\Theta}^{*}=\arg \min _{\boldsymbol{\theta} \in \Omega}\left\{\sup _{\mathbf{x} \in U_{1}, \hat{\mathbf{x}} \mathrm{U}_{2}}\left|f_{1}(\overline{\mathbf{x}})-\hat{f}_{1}(\hat{\mathbf{x}} \mid \boldsymbol{\Theta})\right|\right\}
$$

and neural network approximation error $\delta_{n}$

$$
\delta_{n}(\mathbf{x}, \hat{\mathbf{x}})=f_{1}(\overline{\mathbf{x}})-\hat{f}_{1}\left(\hat{\mathbf{x}} \mid \Theta^{*}\right)
$$

Here, $\boldsymbol{\Theta}$ denotes the estimation of $\boldsymbol{\Theta}^{*}$ and let $\widetilde{\boldsymbol{\Theta}}=\boldsymbol{\Theta}-\boldsymbol{\Theta}^{*}$.

Assumption 3. For the given positive-definite matrices $\mathbf{Q}_{1}$ and $\mathbf{Q}_{2}$, there exist positive-definite solutions $\mathbf{P}_{1}$ and $\mathbf{P}_{2}$ for the matrix equations

$$
\begin{gathered}
\left(\mathbf{A}-\mathbf{B} \mathbf{K}_{\mathrm{c}}^{\mathrm{T}}\right)^{\mathrm{T}} \mathbf{P}_{1}+\mathbf{P}_{1}\left(\mathbf{A}-\mathbf{B} \mathbf{K}_{\mathrm{c}}^{\mathrm{T}}\right)+\mathbf{Q}_{1}=\mathbf{0} \\
\left(\mathbf{A}-\mathbf{K}_{o} \mathbf{C}^{\mathrm{T}}\right)^{\mathrm{T}} \mathbf{P}_{2}+\mathbf{P}_{2}\left(\mathbf{A}-\mathbf{K}_{o} \mathbf{C}^{\mathrm{T}}\right)+\mathbf{Q}_{2}=\mathbf{0} \\
\mathbf{P}_{2} \mathbf{B}_{c}=\mathbf{C}
\end{gathered}
$$

The neural network controller is designed as

$$
u=N(\varsigma)\left[-\mathbf{\Theta} \xi(\hat{\mathbf{x}})+y_{m}^{n}-\mathbf{K}_{\mathrm{c}}^{\mathrm{T}} \hat{\mathbf{e}}+u_{a}+u_{s}\right]
$$

with

$$
\begin{aligned}
& u_{a}=-\mathbf{K}_{\mathrm{o}}^{\mathrm{T}} \mathbf{P}_{1} \hat{\mathbf{e}} \\
& u_{s}=-\mathrm{k} \operatorname{sgn}\left(\mathbf{B}_{\mathrm{c}}^{\mathrm{T}} \mathbf{P}_{2} \widetilde{\mathbf{e}}\right) \\
& \dot{\boldsymbol{\zeta}}=\widetilde{e}_{1}^{\mathrm{T}} L^{-1}(s)\left[\boldsymbol{\Theta} \xi(\hat{\mathbf{x}})-y_{m}^{n}+\mathbf{K}_{\mathrm{c}}^{\mathrm{T}} \hat{\mathbf{e}}-u_{a}-u_{s}\right]
\end{aligned}
$$

and the following adaptation law as

$$
\dot{\boldsymbol{\Theta}}=\gamma \mathbf{B}_{\mathrm{c}}^{\mathrm{T}} \mathbf{P}_{2} \widetilde{\mathbf{e}} L^{-1}(s) \xi^{T}(\hat{\mathbf{x}})
$$

where $u_{a}$ is the feedback control for $\hat{\mathbf{e}}, u_{s}$ is a controller to compensate neural network approximation error and external disturbances and $\gamma>0$ is the adaptation gain to be designed.
Assumption 4. $\delta_{m}=L^{-1}(s)\left(\delta_{n}(\mathbf{x}, \hat{\mathbf{x}})+d(t)+u_{a}+u_{s}\right)-u_{a}-u_{s}$ is bounded i.e. $\delta_{m}$ satisfies $\left\|\delta_{m}\right\|<\delta$.

The main property of indirect adaptive neural network control scheme is summarized in the following theorem.

Theorem 1. For the nonlinear SISO system (1), the neural controller law is chosen as (29)-(30) with the adaptation law (31). If Assumptions 1-4 are satisfied, then the proposed neural control scheme can guarantee the following properties:

(1) The whole closed-loop is stable, i.e., $\mathbf{x}, \hat{\mathbf{x}}, \mathbf{e}, \hat{\mathbf{e}}, u \in L_{\infty}$

(2) $\lim _{t \rightarrow \infty} e_{1}=0$

Proof. Consider the Lyapunov function candidate

$$
\mathrm{V}=\frac{1}{2} \hat{\mathbf{e}}^{\mathrm{T}} \mathbf{P}_{1} \hat{\mathbf{e}}+\frac{1}{2} \widetilde{\mathbf{e}}^{\mathrm{T}} \mathbf{P}_{2} \widetilde{\mathbf{e}}+\frac{1}{2 \gamma} \widetilde{\boldsymbol{\Theta}} \widetilde{\boldsymbol{\Theta}}^{\mathrm{T}}
$$

The time derivative of $\mathrm{V}$ is

$$
\begin{aligned}
\dot{\mathrm{V}} & =\frac{1}{2} \dot{\hat{\mathbf{e}}}^{\mathrm{T}} \mathbf{P}_{1} \hat{\mathbf{e}}+\frac{1}{2} \hat{\mathbf{e}}^{\mathrm{T}} \mathbf{P}_{1} \dot{\hat{\mathbf{e}}}+\frac{1}{2} \dot{\overrightarrow{\mathbf{e}}}^{\mathrm{T}} \mathbf{P}_{2} \widetilde{\mathbf{e}} \\
& +\frac{1}{2} \widetilde{\mathbf{e}}^{\mathrm{T}} \mathbf{P}_{2} \dot{\tilde{\mathbf{e}}}+\frac{1}{\gamma} \widetilde{\boldsymbol{\Theta}} \dot{\widetilde{\mathbf{\Theta}}}^{\mathrm{T}}
\end{aligned}
$$

Substituting (12) and (20) into (33) yields

$$
\begin{aligned}
\dot{\mathrm{V}} & =\frac{1}{2} \hat{\mathbf{e}}^{\mathrm{T}}\left[\left(\mathbf{A}-\mathbf{B} \mathbf{K}_{\mathrm{c}}^{\mathrm{T}}\right)^{\mathrm{T}} \mathbf{P}_{1}+\mathbf{P}_{1}\left(\mathbf{A}-\mathbf{B} \mathbf{K}_{\mathrm{c}}^{\mathrm{T}}\right)\right] \hat{\mathbf{e}}+\hat{\mathbf{e}}^{\mathrm{T}} \mathbf{P}_{1} \mathbf{K}_{o} \mathbf{C}^{\mathrm{T}} \widetilde{\mathbf{e}} \\
& +\frac{1}{2} \widetilde{\mathbf{e}}^{\mathrm{T}}\left[\left(\mathbf{A}-\mathbf{K}_{o} \mathbf{C}^{\mathrm{T}}\right)^{\mathrm{T}} \mathbf{P}_{2}+\mathbf{P}_{2}\left(\mathbf{A}-\mathbf{K}_{o} \mathbf{C}^{\mathrm{T}}\right)\right] \widetilde{\mathbf{e}} \\
& +\widetilde{\mathbf{e}}^{\mathrm{T}} \mathbf{P}_{2} \mathbf{B}_{c}\left[L^{-1}(s) f_{1}(\overline{\mathbf{x}})+L^{-1}(s)\left(\mathbf{K}_{\mathrm{c}}^{\mathrm{T}} \hat{\mathbf{e}}-y_{m}^{n}\right)\right. \\
& \left.+L^{-1}(s) d(t)-L^{-1}(s) g_{0} u\right]+\frac{1}{\gamma} \widetilde{\boldsymbol{\Theta}} \dot{\widetilde{\mathbf{\Theta}}}^{\mathrm{T}}
\end{aligned}
$$

Then by using (27), and (28) we can write (34) as

$$
\begin{aligned}
\dot{\mathrm{V}} & =-\frac{1}{2} \hat{\mathbf{e}}^{\mathrm{T}} \mathbf{Q}_{1} \hat{\mathbf{e}}+\hat{\mathbf{e}}^{\mathrm{T}} \mathbf{P}_{1} \mathbf{K}_{o} \mathbf{C}^{\mathrm{T}} \widetilde{\mathbf{e}}-\frac{1}{2} \widetilde{\mathbf{e}}^{\mathrm{T}} \mathbf{Q}_{2} \widetilde{\mathbf{e}} \\
& +\widetilde{\mathbf{e}}^{\mathrm{T}} \mathbf{P}_{2} \mathbf{B}_{c}\left[L^{-1}(s) f_{1}(\overline{\mathbf{x}})+L^{-1}(s)\left(\mathbf{K}_{\mathrm{c}}^{\mathrm{T}} \hat{\mathbf{e}}-y_{m}^{n}\right)\right. \\
& \left.+L^{-1}(s) d(t)-L^{-1}(s) g_{0} u\right]+\frac{1}{\gamma} \widetilde{\tilde{\Theta}^{\mathrm{\Theta}}}
\end{aligned}
$$

Using (22) and (26), (35) can be further written as

$$
\begin{aligned}
\dot{\mathrm{V}} & =-\frac{1}{2} \hat{\mathbf{e}}^{\mathrm{T}} \mathbf{Q}_{1} \hat{\mathbf{e}}+\hat{\mathbf{e}}^{\mathrm{T}} \mathbf{P}_{1} \mathbf{K}_{o} \mathbf{C}^{\mathrm{T}} \widetilde{\mathbf{e}}-\frac{1}{2} \widetilde{\mathbf{e}}^{\mathrm{T}} \mathbf{Q}_{2} \widetilde{\mathbf{e}} \\
& +\widetilde{\mathbf{e}}^{\mathrm{T}} \mathbf{P}_{2} \mathbf{B}_{c}\left[-L^{-1}(s) \widetilde{\boldsymbol{\Theta}} \xi(\hat{\mathbf{x}})+L^{-1}(s) \boldsymbol{\Theta} \xi(\hat{\mathbf{x}})\right. \\
& +L^{-1}(s) \delta_{f}(\mathbf{x}, \hat{\mathbf{x}})+\left(u_{a}+u_{s}\right)-\left(u_{a}+u_{s}\right) \\
& \left.+L^{-1}(s)\left(\mathbf{K}_{\mathrm{c}}^{\mathrm{T}} \hat{e}-y_{m}^{n}\right)+L^{-1}(s) d(t)-L^{-1}(s) g_{0} u\right] \\
& +\frac{1}{\gamma} \widetilde{\boldsymbol{\Theta}} \dot{\widetilde{\boldsymbol{\Theta}}}^{\mathrm{T}}
\end{aligned}
$$

After substituting (29) and (30) and adaptation law (31) in (36) and using Assumption 4 we have 


$$
\begin{aligned}
\dot{\mathrm{V}} & =-\frac{1}{2} \hat{\mathbf{e}}^{\mathrm{T}} \mathbf{Q}_{1} \hat{\mathbf{e}}-\frac{1}{2} \widetilde{\mathbf{e}}^{\mathrm{T}} \mathbf{Q}_{2} \widetilde{\mathbf{e}}+\widetilde{\mathbf{e}}^{\mathrm{T}} \mathbf{P}_{2} \mathbf{B}_{c} \delta_{m}+\widetilde{\mathbf{e}}^{\mathrm{T}} \mathbf{P}_{2} \mathbf{B}_{c} u_{s} \\
& +\widetilde{\mathbf{e}}^{\mathrm{T}} \mathbf{P}_{2} \mathbf{B}_{c} L^{-1}(s)\left(\boldsymbol{\Theta} \xi(\hat{\mathbf{x}})-y_{m}^{n}+\mathbf{K}_{\mathrm{c}}^{\mathrm{T}} \hat{e}-u_{a}-u_{s}\right)\left(1+g_{0} N(\varsigma)\right) \\
& =-\frac{1}{2} \hat{\mathbf{e}}^{\mathrm{T}} \mathbf{Q}_{1} \hat{\mathbf{e}}-\frac{1}{2} \widetilde{\mathbf{e}}^{\mathrm{T}} \mathbf{Q}_{2} \widetilde{\mathbf{e}}+\widetilde{\mathbf{e}}^{\mathrm{T}} \mathbf{P}_{2} \mathbf{B}_{c}\left(\delta_{m}+u_{s}\right)+\left(1+g_{0} N(\varsigma)\right) \dot{\zeta}
\end{aligned}
$$

Substituting $u_{s}$ into (37) yields:

$$
\dot{\mathrm{V}}=-\frac{1}{2} \hat{\mathbf{e}}^{\mathrm{T}} \mathbf{Q}_{1} \hat{\mathbf{e}}-\frac{1}{2} \widetilde{\mathbf{e}}^{\mathrm{T}} \mathbf{Q}_{2} \widetilde{\mathbf{e}}+(\delta-\mathrm{k})\left(\tilde{\mathbf{e}}^{\mathrm{T}} \mathbf{P}_{2} \mathbf{B}_{c}\right)+\left(1+g_{0} N(\varsigma)\right) \dot{\zeta}
$$

Choosing $\mathrm{k} \geq \delta$, we have:

$$
\dot{\mathrm{V}} \leq-\frac{1}{2} \hat{\mathbf{e}}^{\mathrm{T}} \mathbf{Q}_{1} \hat{\mathbf{e}}-\frac{1}{2} \widetilde{\mathbf{e}}^{\mathrm{T}} \mathbf{Q}_{2} \widetilde{\mathbf{e}}+\left(1+g_{0} N(\varsigma)\right) \dot{\zeta}
$$

Denoting $\mathbf{Q}=\operatorname{diag}\left[\mathbf{Q}_{1}, \mathbf{Q}_{2}\right]$ and $\mathbf{E}^{\mathrm{T}}=\left[\hat{\mathbf{e}}^{\mathrm{T}}, \widetilde{\mathbf{e}}^{\mathrm{T}}\right]$, (39) becomes

$$
\dot{\mathrm{V}} \leq-\frac{1}{2} \mathbf{E}^{\mathrm{T}} \mathbf{Q E}+\left(1+g_{0} N(\varsigma)\right) \dot{\zeta}
$$

Integrating (40) over $\left\lfloor 0, t_{f}\right\rfloor$, we have

$$
\mathrm{V}(t) \leq \mathrm{V}\left(t_{0}\right)+\int_{0}^{t_{f}}\left(1+g_{0} N(\varsigma)\right) \dot{\zeta} d \tau
$$

According to Lemma 1, it can be concluded from (41) that $\mathrm{V}(t), \int_{0}^{t_{f}}\left(1+g_{0} N(\varsigma)\right) \dot{\zeta} d \tau$ and $\varsigma(t)$ are bounded in $\left\lfloor 0, t_{f}\right)$.

We know that the above discussion is also true for $t_{f}=\infty$

(Boulkroune et al. 2010). Since $\int_{0}^{t_{f}}\left(1+g_{0} N(\varsigma)\right) \dot{\zeta} d \tau$ is bounded, it is concluded that $\mathbf{e}, \hat{\mathbf{e}}, \mathbf{x}, \hat{\mathbf{x}}, u \in L_{\infty}$ and $\lim _{t \rightarrow \infty} \mathbf{E}=\mathbf{0}$. Thus, $\lim _{t \rightarrow \infty} \tilde{\mathbf{e}}=\mathbf{0}$ and $\lim _{t \rightarrow \infty} \hat{\mathbf{e}}=\mathbf{0}$. Since $\quad \mathbf{e}=\hat{\mathbf{e}}+\widetilde{\mathbf{e}} \quad$ and $e_{1}=\mathbf{C}^{\mathrm{T}} \mathbf{e}, \quad \lim _{t \rightarrow \infty} e_{1}=0$ which completes the proof.

Remark 3. Since the control law (29) contains the sign function, direct application of such control may result in chattering caused by the signal discontinuity. In order to remedy the chattering phenomenon, the sign function is replaced by saturation function with a boundary layer $\varphi$ of the form

$$
\operatorname{sat}\left(\frac{\left(\widetilde{\mathbf{e}}^{\mathrm{T}} \mathbf{P}_{2} \mathbf{B}_{c}\right)}{\varphi}\right)= \begin{cases}\operatorname{sgn}\left(\frac{\left(\widetilde{\mathbf{e}}^{\mathrm{T}} \mathbf{P}_{2} \mathbf{B}_{c}\right)}{\varphi}\right), & \left|\frac{\left(\tilde{\mathbf{e}}^{\mathrm{T}} \mathbf{P}_{2} \mathbf{B}_{c}\right)}{\varphi}\right|>1 \\ \frac{\left(\widetilde{\mathbf{e}}^{\mathrm{T}} \mathbf{P}_{2} \mathbf{B}_{c}\right)}{\varphi}, & \left|\frac{\left(\widetilde{\mathbf{e}}^{\mathrm{T}} \mathbf{P}_{2} \mathbf{B}_{c}\right)}{\varphi}\right| \leq 1\end{cases}
$$

Remark 4. Since (26) is only guaranteed within a certain compact set, the stability results in this paper are semi-global which means for any compact set, there exists a controller with neural approximation with sufficiently large hidden nodes such that when the initial states are within this set, all the closed-loop signals are bounded. In realistic applications due to computational problem, the hidden nodes usually cannot be chosen too large which implies that the neural system approximation ability is limited and some constraints on compact set are necessary to guarantee such an approximation.

\section{SIMULATION RESULTS}

In this section, the simulation study is carried out to show the proficiency of the proposed adaptive neural controller. Consider an academic SISO non-affine nonlinear system (Liu and Wang 2009). The dynamic equations of such system are given by

$$
\begin{aligned}
& \dot{x}_{1}=x_{2} \\
& \dot{x}_{2}=-x_{1}+2 x_{2}+\frac{u}{\sqrt{|u|+0.1}}-2 x_{1}^{2} x_{2}+d(t)
\end{aligned}
$$

$y=x_{1}$

The control objective is to force the system output $x_{1}$ to track the sinusoidal desired trajectory $y_{m}=0.5 \sin (t)$. The external disturbances $d_{1}(t)=0.5 \sin (2 t)$ is added to the system (37). The design parameters are chosen as $\gamma=10^{3}, \mathrm{k}=20, \varphi=0.001$. The feedback and observer gain matrices are given by

$$
\mathbf{K}_{\mathrm{c}}=\left[\begin{array}{ll}
144 & 24
\end{array}\right]^{\mathrm{T}}, \mathbf{K}_{\mathrm{o}}=\left[\begin{array}{ll}
60 & 900
\end{array}\right]^{\mathrm{T}} .
$$

In order to use the SPR-Lyapunov design approach, $L_{1}^{-1}(s)$ is chosen as $L_{1}^{-1}(s)=\frac{1}{s+2}$. In addition, the positive-definite matrices $\mathbf{Q}_{1}$, and $\mathbf{Q}_{2}$ are chosen as

$$
\mathbf{Q}_{1}=\left[\begin{array}{cc}
10^{-2} & 0 \\
0 & 10^{-2}
\end{array}\right], \mathbf{Q}_{2}=\left[\begin{array}{cc}
10 & 0 \\
0 & 10
\end{array}\right]
$$

In this simulation we take $l=9$ nodes to construct the neural network approximator with centers evenly distributed in $\left[\begin{array}{ll}\hat{x}_{1} & \hat{x}_{2}\end{array}\right] \in[-2,2] \times[-2,2]$, the widths are chosen as $\eta=1.8$, and the initial parameters matrix of neural network is $\boldsymbol{\Theta}_{0}=\mathbf{0}$. The simulation is carried out with initial conditions $\mathbf{x}_{0}=[-0.2,0.4]^{\mathrm{T}}$, and $\hat{\mathbf{e}}_{0}=[0.1,0.1]^{\mathrm{T}}$. Fig 1 shows the tracking performance of the proposed controller. We can see that the tracking error is bounded and the designed adaptive neural network output feedback controller can effectively achieve the trajectory tracking of the plant.

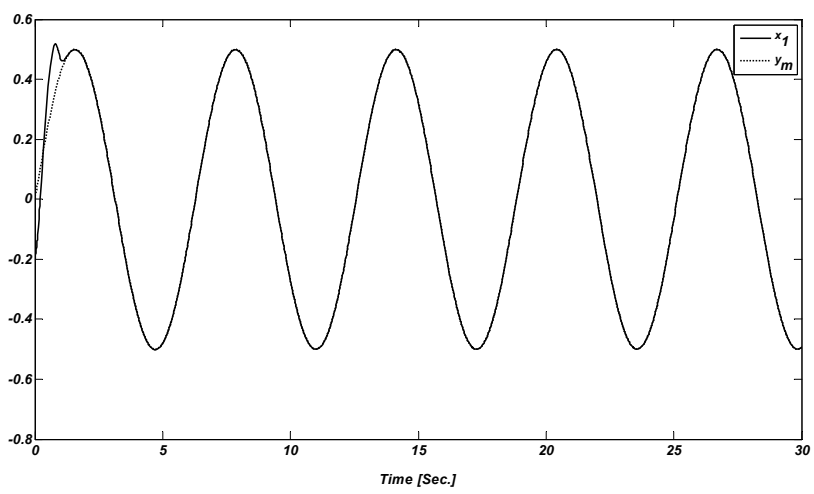

Fig. 1. Trajectories of output variable and reference signal by proposed observer-based neural adaptive controller. 
Fig 2 shows the control signal and it is illustrated that the control signal is bounded. The boundedness of the neural network parameters is illustrated in Fig 3. The variation of the adaptive parameter $\varsigma$ and Nussbaum gain $N(\varsigma)$ is shown in Fig 4. From these figures, we can see that all signals are bounded.

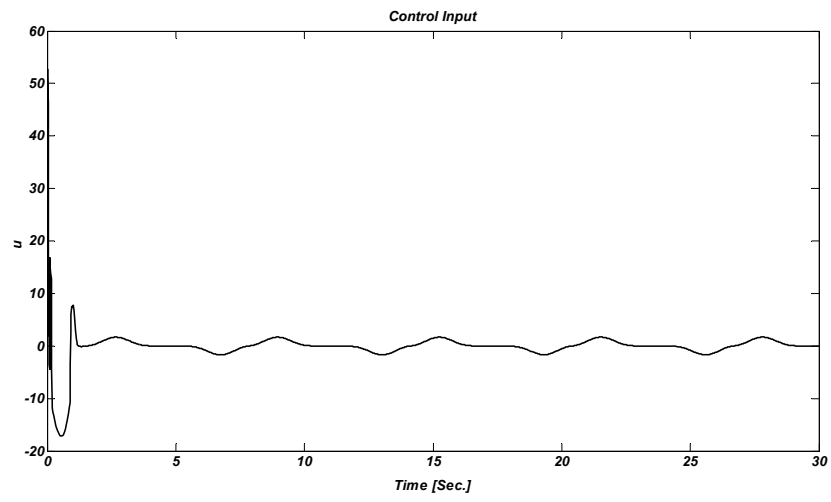

Fig. 2. Control input $u$.

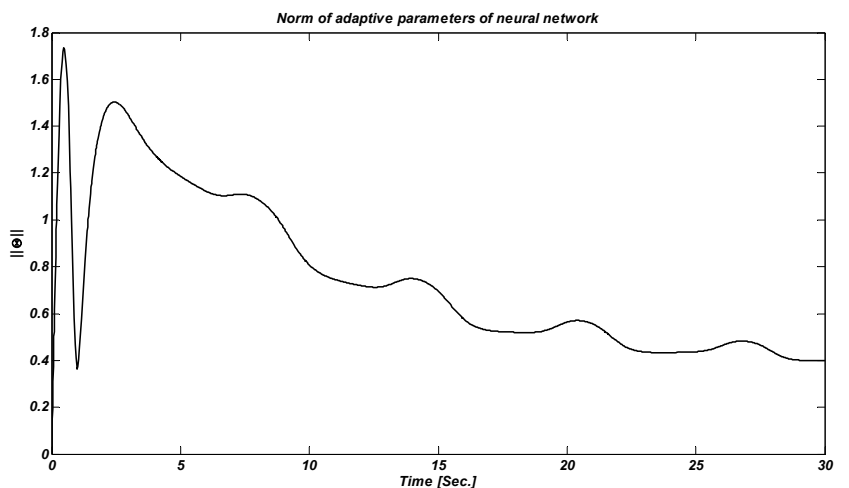

Fig. 3. The norm of adaptive parameters $\|\boldsymbol{\Theta}\|$.

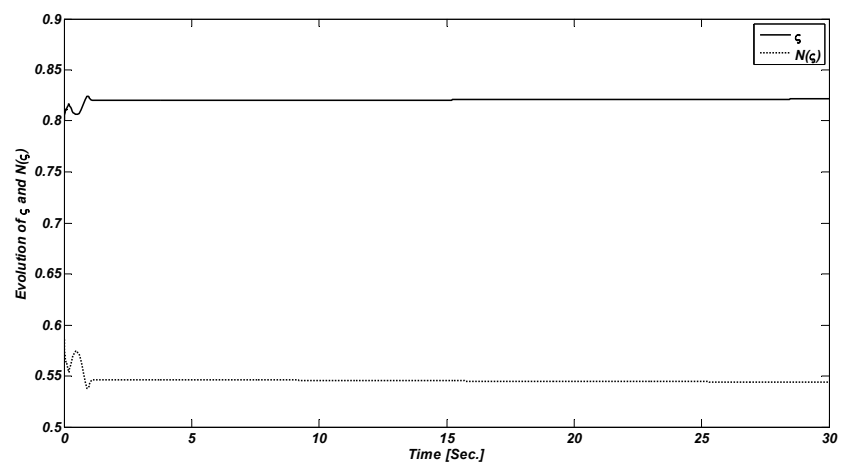

Fig. 4. The adaptive parameter $\varsigma$ and Nussbaum gain $N(\varsigma)$.

\section{CONCLUSION}

In this paper, an adaptive output feedback neural network (NN) control has been investigated for a class of SISO nonaffine nonlinear system with unknown control gain, using Lyapunov method combining with the Mean Value Theorem and Nussbaum gain technique. In this approach, it does not require to assume that the system states are measurable. Thus an observer was designed to estimate unmeasured states. It has been proven that the proposed adaptive observer-based controller can guarantee the convergence of the tracking errors to zero and the boundedness of all the signals in the closed-loop system. The simulation example showed the effectiveness of the proposed approach.

\section{REFERENCES}

Boulkroune, A., Tadjine, M., Saad, M.M., Farza, M. (2010). Fuzzy adaptive controller for MIMO nonlinear systems with known and unknown control direction. Fuzzy Sets and Systems, 161, 797-820.

Chang, Y.C. (2000). Robust tracking control for nonlinear MIMO systems via fuzzy approaches. Automatica, 36, 1535-1545.

Du, H, Chen, X. (2009). NN-based output feedback adaptive variable structure control for a class of non-affine nonlinear systems: A nonseparation principle design. Neurocomputing, 72, 2009-2016.

Frikha, S., Djemel, M., Derbel, N. (2010). Observer-based adaptive neuro-sliding mode control for MIMO nonlinear systems. International Journal of Control, Automation, and systems, 8 (2), 257-265.

Khalil, HK. (2002). Nonlinear systems. 3rd Ed., Prentice Hall, NJ.

Labiod, S., Guerra, T.M. (2007). Direct adaptive fuzzy control for a class of MIMO nonlinear systems. International Journal of System Science, 38 (8), 665-675.

Labiod, S., Guerra, T.M. (2007). Adaptive fuzzy control of a class of SISO non-affine nonlinear systems. Fuzzy Sets and Systems, 158 (10), 1126-1137.

Leu, Y.G., Lee, T.T., Wang, W.Y. (1999). Observer-based adaptive fuzzy-neural control for unknown nonlinear dynamical systems. IEEE Transactions on Systems, Man, and Cybernetics-Part B: Cybernetics, 29 (5), 583-591.

Li, H.X., Tong, S. (2003). A hybrid adaptive fuzzy control for a class of nonlinear MIMO systems. IEEE Transactions on Fuzzy Systems, 11 (1), 24-34.

Lian, J., and Zak, S.H. (2009). Control of uncertain systems. Nof, S.Y. (ed.) Springer Handbook of Automation, Springer.

Liu, Y.J., Tong, S.C., Wang, W., Li, Y.M. (2009). Observer-based direct adaptive fuzzy control of uncertain nonlinear systems and its applications. International Journal of Control, Automation, and systems, 7 (4), 681-690.

Liu, Y.J., Zheng, Y.Q. (2009). Adaptive fuzzy tracking control for a class of uncertain nonlinear systems. Chinese Control and Decision Conference, 941-944.

Liu, Y.J., Zheng, Y.Q. (2009). Adaptive-robust fuzzy control for a class of uncertain chaotic systems. Nonlinear Dynamics, 57, 431-439.

Liu, Y.J., Wang, Z.F. (2009). Adaptive fuzzy controller design of nonlinear systems with unknown gain sign. Nonlinear Dynamics, 58, 687-695.

Liu, Y.J., Li, Y.X. Adaptive fuzzy output-feedback control of uncertain SISO nonlinear systems. Nonlinear Dynamics, DOI: 10.1007/s11071-010-9684-8.

Nussbaum, R.D. (1983). Some remarks on the conjecture in parameter adaptive control. Systems and Control Letters, 3, 243246.

Ordonez, R., Passino, K.M. (1999). Stable multi-input multi-output adaptive fuzzy/neural control. IEEE Transactions on Fuzzy Systems, 4, 339-359.

Park, J.H., Huh, S.H., Kim, S.H., Seo, S.J., Park, G.T. (2005). Direct adaptive controller for non-affine nonlinear systems using selfstructuring neural networks, IEEE Transactions on Neural Networks, 16 (2), 414-422.

Shaocheng, T., Bin, C., Yongfu, W. (2005). Fuzzy adaptive output feedback control for MIMO nonlinear systems. Fuzzy Sets and Systems, 156, 285-299.

Xudong, Y., Jingping, J. (1998). Adaptive nonlinear design without a priori knowledge of control directions. IEEE Transactions on Automatic Control, 43 (11), 1617-1621.

Ye, XD. (2001). Adaptive nonlinear output-feedback control with unknown high-frequency gain sign. IEEE Transactions on Automatic Control, 46 (1), 112-115. 